A fluorescence assay for detecting amyloid- $\beta$ using the cytomegalovirus (CMV) enhancer/promoter

Zachary M. Carrico, Geneva Le, Roberto Malinow

Supplementary information:

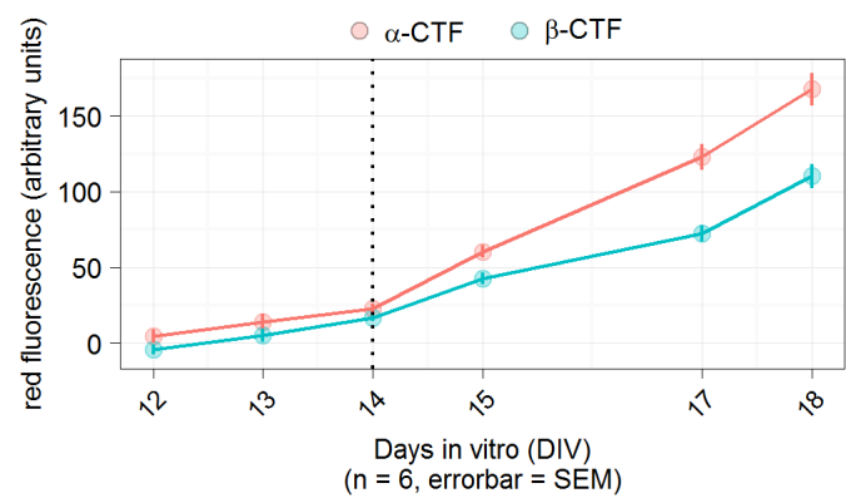

Supplementary Information Figure 1. AAV2(CMV:tdTomato) shows a decrease in fluorescence in response to overexpressed $\boldsymbol{\alpha}$-CTF relative to $\boldsymbol{\beta}$-CTF. Primary neuronal cultures grown in 384 -well plates were infected with reporter-containing AAV virus added at 9 DIV. $\beta$ - or $\alpha$-secretase carboxyl terminal products $(\beta-\mathrm{CTF}$ or $\alpha-\mathrm{CTF})$ of amyloid-precursor protein (APP) were overexpressed using Sindbis virus infections at 14 DIV. $\beta$-CTF leads to production of A $\beta$, while $\alpha$-CTF does not and thus serves as a negative control. This method of detecting A $\beta$ is noteworthy, but was not pursued further because synthetic $A \beta$ peptide was more experimentally tractable. $n$ indicates number of wells used for each condition and the error-bars are standard-error of the mean (SEM).

\title{
General reagents/conditions:
}

Unless otherwise stated, all general chemicals were from Sigma-Aldrich, and all neuroactive reagents from Tocris Bioscience. All procedures involving animals were approved by the Institutional Animal Care and Use Committees of the University of California, San Diego.

\section{Primary neuronal cultures}

Primary hippocampal neurons were made according to previously described protocols with minor modifications [1,2]. Hippocampi from P0-P2 Sprague-Dawley rat pups were first dissected in ice-cold dissection media (in mM: $82 \mathrm{Na} 2 \mathrm{SO} 4,30 \mathrm{~K} 2 \mathrm{SO} 4,5.8 \mathrm{MgCl} 2,0.25 \mathrm{CaCl}$, 8 glucose, 1 HEPES buffer; $2 \%$ Phenol Red solution) before being cut into fine pieces using a scalpel. The hippocampal tissue was then resuspended in dissociation media (dissection media supplemented with $2 \mathrm{mM}$ L-cysteine hydrochloride, 10-20 units papain, pH adjusted to 7.4) and further dissociated using a serological pipette. The suspension was left at room temperature for 30 minutes with gentle rocking. $1 \mu \mathrm{L}$ DNaseI was added to digest precipitated DNA from dead cells. Neurons were then filtered through a $70 \mu \mathrm{m}$ cell strainer to remove undissociated tissue and spun at $1000 \mathrm{x} g$. After removal of most of the supernatant the neurons were resuspended in plating media (Neurobasal-A, 10\% FBS, $0.5 \%$ Pen/Strep and $0.25 \%$ Glutamax) 
and counted. $45 \mu \mathrm{L}$ of $35000-50000$ neurons/ml were added to each well of Corning Bio-Coat poly-D-lysine flat-bottomed clear 384-well plates (Corning part number 3845). After $1 \mathrm{~h}$ at 37 ${ }^{\circ} \mathrm{C}$ and $5 \% \mathrm{CO}_{2}, 45 \mathrm{uL}$ of reduced FBS media was added (Neurobasal-A, 5\% FBS, 2\% B-27, $0.5 \%$ Pen/Strep, $0.25 \%$ Glutamax) was added. Thereafter, half the media was replaced each time (Neurobasal-A, 2\% B-27, 0.5\% Pen/Strep, 0.25\% Glutamax) beginning on day 2, day 5 (along with $10 \mu \mathrm{M}$ Cytosine $\beta$-D-arabinofuranoside to inhibit non-neuronal replication), and every 2-4 days afterwards

\section{References:}

1. Aow J, Dore K, Malinow R (2015) Conformational signaling required for synaptic plasticity by the NMDA receptor complex. Proc Natl Acad Sci U S A 112: 14711-14716.

2. Dore K, Aow J, Malinow R (2015) Agonist binding to the NMDA receptor drives movement of its cytoplasmic domain without ion flow. Proc Natl Acad Sci U S A 112: 1470514710. 